\title{
Statistical validation of individual fibre segmentation from tomograms and microscopy
}

Emerson, Monica Jane; Dahl, Vedrana Andersen; Conradsen, Knut; Mikkelsen, Lars Pilgaard; Dahl, Anders Bjorholm

Published in:

Composites Science and Technology

Link to article, DOI:

10.1016/j.compscitech.2018.03.027

Publication date:

2018

Document Version

Peer reviewed version

Link back to DTU Orbit

Citation (APA):

Emerson, M. J., Dahl, V. A., Conradsen, K., Mikkelsen, L. P., \& Dahl, A. B. (2018). Statistical validation of individual fibre segmentation from tomograms and microscopy. Composites Science and Technology, 160, 208215. https://doi.org/10.1016/j.compscitech.2018.03.027

\section{General rights}

Copyright and moral rights for the publications made accessible in the public portal are retained by the authors and/or other copyright owners and it is a condition of accessing publications that users recognise and abide by the legal requirements associated with these rights.

- Users may download and print one copy of any publication from the public portal for the purpose of private study or research.

- You may not further distribute the material or use it for any profit-making activity or commercial gain

- You may freely distribute the URL identifying the publication in the public portal 


\title{
Statistical Validation of Individual Fibre Segmentation from Tomograms and Microscopy
}

\author{
Monica Jane Emerson ${ }^{\mathrm{a}, *}$, Vedrana Andersen Dahl ${ }^{\mathrm{b}}$, Knut Conradsen ${ }^{\mathrm{b}}$, Lars \\ Pilgaard Mikkelsen ${ }^{\mathrm{a}}$, Anders Bjorholm Dahl ${ }^{\mathrm{a}}$ \\ ${ }^{a}$ Department of Applied Mathematics and Computer Science, Technical University of \\ Denmark, Denmark. \\ ${ }^{b}$ Department of Wind Energy, Technical University of Denmark, Denmark.
}

\begin{abstract}
Imaging with X-ray computed tomography (CT) enables non-destructive 3D characterisations of the micro-structure inside fibre composites. In this paper we validate the use of X-ray $\mathrm{CT}$ coupled with image analysis for characterising unidirectional (UD) fibre composites. We compare X-ray CT at different resolutions to optical microscopy and scanning electron microscopy, where we characterise fibres by their diameters and positions. In addition to comparing individual fibre diameters, we also model their spatial distribution, and compare the obtained model parameters. Our study shows that X-ray CT is a high precision technique for characterising fibre composites and, with our suggested image analysis method for fibre detection, high precision is also obtained at low resolutions. This has great potential, since it allows larger fields of view to be analysed. Besides analysing representative volumes with high precision, we demonstrate that based on our methodology for individual fibre segmentation it is now possible to study complete bundles at the fibre scale and reveal inhomogeneities in the physical sample.
\end{abstract}

Keywords: Geometrical characterisation A. Polymer-matrix composites (PMCs) A. Glass fibres C. Statistics D. Non-destructive testing

\footnotetext{
${ }^{*}$ Corresponding author

Email address: monj@dtu.dk (Monica Jane Emerson)
} 


\section{Introduction}

Imaging is an important method for characterising the micro-structure of materials. Characterising a material requires measurements of the size and shape of material features, and this often involves an automated image analysis 5 method. Therefore, the image-based characterisation of a material's microstructure can be seen as a coupling of the imaging system and the image analysis method.

Some imaging modalities, like optical microscopy (OM) and scanning electron microscopy (SEM), directly depict the surface of the imaged sample. Other modalities produce the image indirectly, and X-ray micro-CT (computed tomography) is such a method. Here the attenuation of X-rays penetrating a sample is measured, and a volumetric image is reconstructed from multiple measurements. Since the reconstruction model can introduce artefacts, it is important to determine the accuracy of the measurements obtained from X-ray CT.

X-ray CT has shown to be useful for characterising the micro-structure of fibre composites [1], and the resolution of X-ray CT images allows for studying fibre bundles or individual fibres in 3D [2. Current X-ray micro-CT laboratory scanning systems produce volumetric images of $1000^{3}-2000^{3}$ voxels, often depicting more than 10000 distinguishable fibres, and synchrotron imaging can give volumes containing more voxels. Automated image analysis methods are required to obtain quantitative information from such images.

With sufficient resolution it is possible to characterise individual fibres, which typically involves a segmentation of the fibre to estimate its position and diameter. Individual fibre segmentation poses several challenges. In composites with high fibre volume fractions (FVFs) the fibres are touching, so it can be difficult to distinguish them in an image. Image noise also makes it challenging to automatically segment fibres, and reducing the noise in the image acquisition process requires longer scan times. These challenges become more pronounced when reducing the spatial resolution to include more fibres in the scanned volume. 
X-ray CT imaging systems give some freedom in choosing the spatial resolution of the recorded data, within the limits determined by the scanning setup. However, there is a trade-off between the size of the imaged volume (field of view) and pixel size (spatial resolution) which relates to the size of the small35 est distinguishable features. Increasing the field of view will reduce the spatial resolution. For this reason, it is important to investigate the accuracy of the combined X-ray CT acquisition and analysis pipeline across resolutions.

Assessing the precision of a scanning system can be done using a physical reference object (phantom), with known geometry and material properties, that 40 is similar to the target material. An example of validating the precision of $\mathrm{X}$ ray CT scanning with a physical phantom was shown in [3. Their approach used a simple threshold-based analysis. In our investigation such a phantom should resemble geometry of fibres, and producing such an object is difficult. Alternatively, the size of the fibres can be compared with the results obtained

45 by a system that is more precise than micro-CT, which is why we use OM and SEM.

Employing virtual computerised phantoms, composed of artificially created images, will provide a ground truth reference for the measurements, and this has been used in e.g. 4, 5, 6. Typically, it is difficult to make a virtual phantom that resembles the imaging system accurately enough to assess the precision of the image analysis method over the specific scanning technique, in this case micro-CT scanning. Another method for validating a quantification pipeline (scanning technique and image analysis method) is to compare obtained measures to derived physical parameters, as shown in [7, 8, 9,

Studies for evaluating different tomographic techniques for characterising defects in composites have been performed in [10, 5, 11. These studies focused on porosity in composites, and have been carried out on images where individual fibres are not resolved. Nikishkov et al. [12] also performed void geometry quantification using X-ray $\mathrm{CT}$, and they validated their approach through mi60 croscopy measurements.

Methods for segmenting densely packed UD fibres individually have been 
presented in [13. for a relatively small field of view and in 14] for a much larger field of view. They both carried out finite element simulations based on the segmented fibres. However, these methods were employed for scans of relatively higher resolution with more pixels covering each fibre compared to the capabilities of our method. The average fibre sizes and pixel sizes reported in [13, 14] give fibre diameters around 15 pixels whereas our low-resolution scan has an average diameter of 6 pixels, which allows approximately six times more fibres to be analysed for 2.5 times longer fibres. This significantly larger number of fibres allows a more representative analysis. Segmenting individual fibres with high precision from low-resolution X-ray scans is necessary to facilitate representative and accurate characterisations of the micro-structure inside UD composites.

In [4, 8] we presented a model for fibre segmentation that can handle low75 quality scans containing many fibres. This method can deal with reduced contrast between material phases. Hence, it is robust to noise [15] and, as shown in [8, it is able to segment both glass and carbon UD fibres individually. In the current paper we are now demonstrating that it allows precise segmentation of fibres from low-resolution scans. The method characterises fibres in 3D with fibre "trajectories", centre lines connecting the 2D centre coordinates that belong to the same fibre. In [8] the method was used for measuring individual fibre orientations and relating these to the compression strength of the material. In the current paper we use this segmentation method for estimating the diameter and position of fibres to compare the measurements obtained from micro-CT scans to those obtained from high-resolution SEM and OM images fibre by fibre.

In our validation study, we compare the results obtained from laboratory X-ray CT (XCT) at three resolutions, synchrotron radiation CT (SRCT), OM and SEM images. The fibre diameter measured in pixels in the SRCT and high resolution $\mathrm{XCT}$ is in the same range as the analysis in [13, 14] whereas the midand low-resolution scans have fewer pixels per fibre diameter. This allows us to investigate how the loss in resolution affects the estimates of fibre diameter and position, hereby assessing the degradation of the image analysis methods when 
increasing the imaged field of view.

We employ SEM and OM as reference for the validation. Since SEM and OM images are obtained from a polished surface of the fibre sample, imaged orthogonal to the fibre direction, we can compute the fibre diameters with high precision, by modelling fibres as circles. The OM and SEM images are of very high spatial resolution with between two and fifteen times more pixels per fibre diameter than the X-ray CT scans.

Our proposed validation strategy is based on a statistical analysis of the correlation of the fibre parameters and their spatial distribution obtained using the different scanning modalities and two image analysis methods, our probabilistic algorithm for individual fibre segmentation and the circular Hough transform, that is well suited for recognising circular structures.

\section{Materials}

This study is based on imaging a non-crimp fabric ( $\mathrm{NCF}$ ) glass fibre reinforced polymer. A detailed description of this type of composite is given in [16]. We could have also used other types of unidirectional (UD) composites, such as composites manufactured using filament winding, pultrusion or pre-preg, and even composites based on carbon fibres.

In the specific NCF, glass fibres are arranged in bundles. The load-carrying part of the composite is made of UD fibre bundles that are stitched on backing bundles. Figure 1 shows the sample with the imaged surface marked in green and the imaged volume delineated with a red box. The investigated fibres are from the UD part of the composite. This fibre composite is specified to have approximately 4000 fibres per bundle, an overall fibre volume fraction (FVF) of $57 \%$ and an average fibre diameter of $17 \mu \mathrm{m}$.

For the SEM image to be acquired a thin layer of gold was added to the top of the sample, which was polished away after scanning. The XCT images were obtained by scanning the sample in a laboratory X-ray CT scanner (Carl Zeiss Versa 520) at three different resolutions, and the SRCT scan was acquired at 


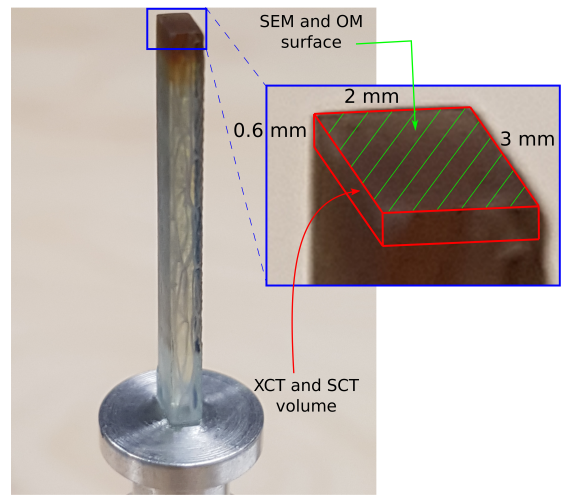

Figure 1: Glass fibre sample mounted for CT scanning. The volume outlined by the red box has been CT-scanned, and the top surface in green has been imaged using SEM and OM.

the synchrotron ESRF (European Synchrotron Radiation Facility, ID19). For $\mathrm{XCT}$ and SRCT the volumetric images were obtained indirectly by computing the image using reconstruction methods. The reconstruction for the lab-based $\mathrm{XCT}$ was performed with the software provided by the scanner supplier and for the SRCT image the filtered back projection algorithm was employed 1

Table 1 lists the pixel/voxel sizes reported by the instruments, which deviate a little from those measured by comparing the images. Therefore, the imagebased measured pixel/voxel sizes relative to the $\mathrm{OM}$ image are also given in Table 1. These were obtained through the image registration process described in Section 3.1 ,

Images from the six scans employed in this study are shown in Fig. 2 after initial alignment of the images. The different images have a very different amount of pixels per fibre, which is easier to see in the digital version of the images or in Fig. 4. The region employed for our validation study contains over 700 fibres in the UD bundle and its size is approximately the size of the images in Fig. 2 , of length $1.02 \mathrm{~mm}$ and width $0.42 \mathrm{~mm}$. Additionally, the distribution of fibre diameters was investigated across the full bundle, covered completely by the

\footnotetext{
${ }^{1}$ The data files for the six scans will be available as a data-in-brief article
} 
Table 1: Instrument reported and measured pixel/voxel sizes for the different scans.

\begin{tabular}{lll}
\hline Scan & Reported & Measured \\
\hline $\mathrm{SEM}$ & $0.1852 \mu \mathrm{m}$ & $0.1882 \mu \mathrm{m}$ \\
$\mathrm{OM}$ & $0.2908 \mu \mathrm{m}$ & (reference) \\
$\mathrm{SRCT}$ & $0.6500 \mu \mathrm{m}$ & $0.6440 \mu \mathrm{m}$ \\
$\mathrm{XCT}_{\mathrm{H}}$ & $1.0376 \mu \mathrm{m}$ & $1.0356 \mu \mathrm{m}$ \\
$\mathrm{XCT}_{\mathrm{M}}$ & $1.6856 \mu \mathrm{m}$ & $1.6835 \mu \mathrm{m}$ \\
$\mathrm{XCT}_{\mathrm{L}}$ & $2.8059 \mu \mathrm{m}$ & $2.8082 \mu \mathrm{m}$ \\
\hline
\end{tabular}

low-resolution X-ray scan, and containing thousands of fibres. The bundle has an approximate length of $3 \mathrm{~mm}$ and a width of $1 \mathrm{~mm}$. For the three-dimensional modalities, 60 slices in a depth of $0.6 \mathrm{~mm}$ were analysed. This depth is shown in Fig. 1 to illustrate how much of the sample was included in the analysis.

\section{Methods}

To get an impression of the 3D structure of the sample, Fig. 3 shows a 3D rendering of the high-resolution XCT scan. Since we have cross-sectional images, fibres are modelled as circles, each of them defined by a centre point and a diameter. These parameters are obtained by image segmentation.

\subsection{Fibre segmentation and matching}

Two image analysis methods were used for extracting fibre parameters, the circular Hough transform [17, 18] and our probabilistic feature labelling method, which we have used for fibre detection in [8, 4].

The circular Hough transform is a two stage algorithm [17, 18]. In the first stage an edge image is computed, and in the second stage circles are matched to the edge image through a voting scheme. The algorithm we use has three 155 parameters including an edge parameter, determining when a pixel is considered an edge pixel, a sensitivity parameter, determining when a circle is detected, and 


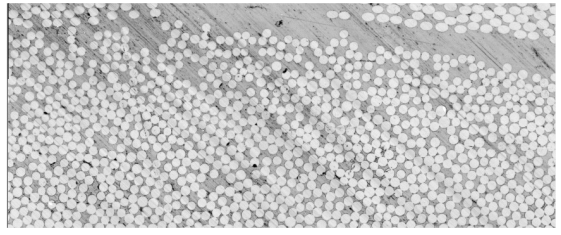

(a) OM image.

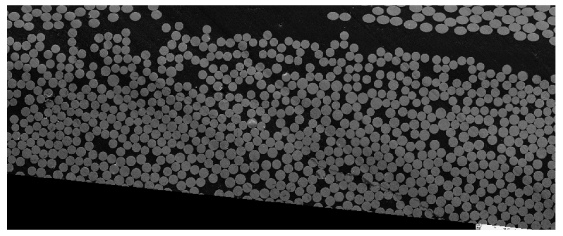

(c) SEM image.

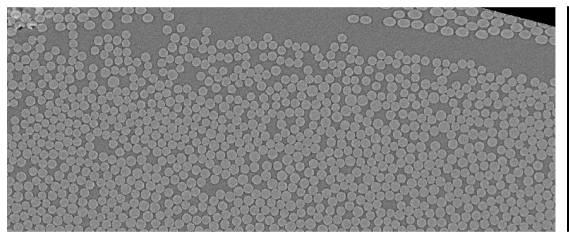

(e) SRCT image.

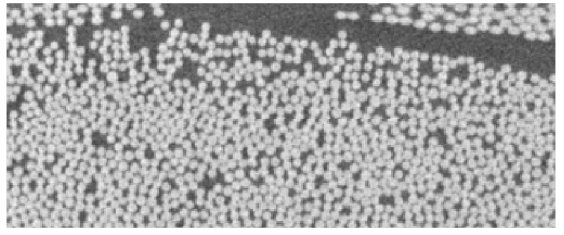

(b) Low resolution XCT image.

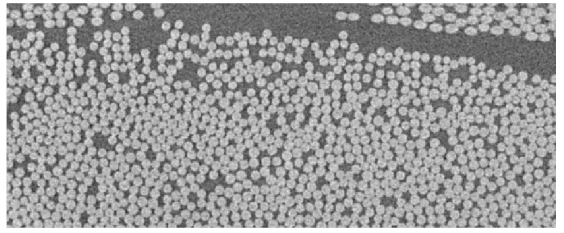

(d) Mid-resolution XCT image.

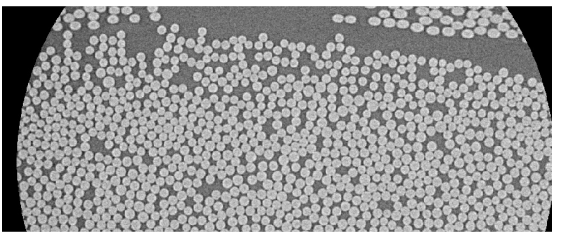

(f) High resolution XCT image.

Figure 2: Aligned images from all six modalities to illustrate the region of interest employed for our validation study. The number of pixels covering each fibre varies significantly between modalities, and this can be better seen in Fig. 4 or in the digital version of the images.

a parameter giving a circle diameter range. In the employed implementation, the diameter range is given as integers, which especially for low resolution images will give a coarse approximation of the fibre diameters. To obtain a higher precision in the diameter estimation, we up-scaled the CT-scanned images using linear interpolation. The SRCT and high-resolution XCT were up-scaled with a factor 2, the mid-resolution XCT with a factor 3 and the low-resolution XCT with a factor 4 . Additionally, the sensitivity parameter was adjusted to find all fibres in the set used for the comparison.

The probabilistic feature labelling is a general method that we have developed for detecting repetitive image features [15, 8, 4]. The method detects repetitive image features through a learning scheme based on limited user input, and here we use it for detecting fibre centre points. This detection returns an 


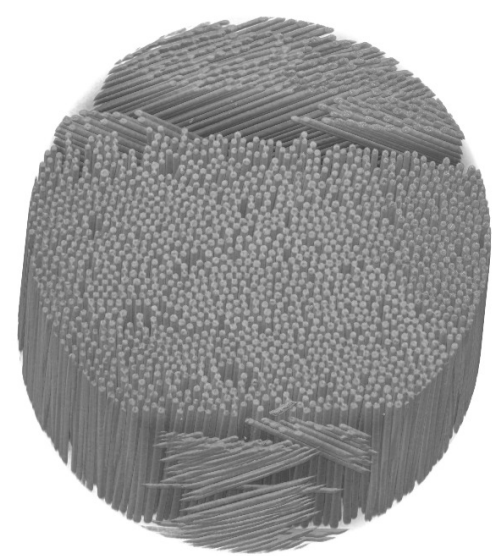

Figure 3: Visualisation of fibres in the high-resolution XCT scan.

image where each pixel has a probability of being a centre point. To compute the centre coordinates, the probability image is thresholded and the average position of each connected component gives the centre position of the fibre.

After detecting the centre points, diameters must be computed. Since the employed UD fibres are very dense, it is reasonable to assume that fibres are in contact. Thus, for our volumetric CT scan, we compute for each fibre a diameter at every $2 \mathrm{D}$ slice as the distance to the closest fibre. Then, we average the $2 \mathrm{D}$ estimations across the 60 slices to obtain a single diameter per fibre. By using the $3 \mathrm{D}$ trajectories to estimate the diameters we give extra robustness to the measurements. However, the averaging can lead to overlapping fibres because fibre trajectories wiggle and thus positions vary across the $2 \mathrm{D}$ slices. For comparing diameter measurements across scanning modalities we use the positions from the top slice for matching corresponding fibres.

For a fibre to fibre comparison, we must establish correspondence between the imaged fibres. Since the OM and SEM images are obtained from the top surface of the sample and the micro-CT images are obtained just beneath this surface, we assume that the relative position of fibres is the same for all modalities. Therefore, the main difference in fibre position between the images can be modelled using a rotation, translation and scale. The transformation is com- 
puted using the model $p=s R q+t$, where $p$ and $q$ are coordinates of corresponding points, $s$ is a scaling factor, $R$ is a rotation matrix and $t$ is a translation. The transformation parameters $s, R$ and $t$ are computed as the least squares fit from four manually annotated points.

After applying the transformation, the fibre parameters across all modalities have a common coordinate system. The final fibre to fibre correspondence is then found as the nearest neighbour using Euclidean distance. Examples of the segmented fibres can be seen in Fig. 4.

\subsection{Statistical analysis}

We consider the fibre diameter measurements by $n=10$ different quantification pipelines (referred to as 'methods' from now on), listed in Tab. 2, Each method consists of two steps: i) scanning technique (called 'mode' from now onward), which refers to a specific imaging modality and resolution, and ii) image analysis method (called 'algorithm' from now onward). The six modes are CT scans including synchrotron, high, medium, and low resolution laboratory X-rays (abbreviated SRCT, $\mathrm{XCT}_{\mathrm{H}}, \mathrm{XCT}_{\mathrm{M}}, \mathrm{XCT}_{\mathrm{L}}$ ) and microscopy images, namely optical and scanning electron microscopy (abbreviated OM and SEM). The algorithms are denoted Prob. for the analysis based on the probabilistic feature labelling in 3D and Hough for the circular Hough transform on the top layer of the sample. We organise the measurements in two different ways: the univariate and the multivariate approach.

Univariate approach. In the univariate approach we investigate the validity of the diameter estimates obtained using the various modes and algorithms. This is done by describing the variation between fibre diameters as deterministic contributions from the methods (or algorithm and modes) adjusting for the individual fibre contributions.

If we want to separate contributions from algorithms and modes we use a three-way factorial design model (Model I), symbolically written Algorithm $\times$ Mode $\times$ Fibre. If we just focus upon the methods (combined effect of algorithm and mode) we use a two-way layout (Model II) written Method $\times$ Fibre. 
Table 2: Model parameters for the univariate approach, statistical Models I and II.

\begin{tabular}{llllllll}
\hline & \multicolumn{4}{c}{ Model I } & \multicolumn{3}{c}{ Model II } \\
& \multicolumn{2}{c}{ Algorithm $\times$ Mode $\times$ Fibre } & \multicolumn{2}{c}{ Method $\times$ Fibre } \\
Abbrev. & Alg., $i=1,2$ & $i$ & $j$ & $D_{i j k}$ & $\nu$ & $D_{\nu k}$ \\
\hline SRCT & Prob. & 1 & 1 & $D_{(1,1, k)}$ & 1 & $D_{(1, k)}$ \\
$\mathrm{XCT}_{\mathrm{H}}$ & Prob. & 1 & 2 & $D_{(1,2, k)}$ & 2 & $D_{(2, k)}$ \\
$\mathrm{XCT}_{\mathrm{M}}$ & Prob. & 1 & 3 & $D_{(1,3, k)}$ & 3 & $D_{(3, k)}$ \\
$\mathrm{XCT}_{\mathrm{L}}$ & Prob. & 1 & 4 & $D_{(1,4, k)}$ & 4 & $D_{(4, k)}$ \\
$\mathrm{SRCT}$ & Hough & 2 & 1 & $D_{(2,1, k)}$ & 5 & $D_{(5, k)}$ \\
$\mathrm{XCT}_{\mathrm{H}}$ & Hough & 2 & 2 & $D_{(2,2, k)}$ & 6 & $D_{(6, k)}$ \\
$\mathrm{XCT}_{\mathrm{M}}$ & Hough & 2 & 3 & $D_{(2,3, k)}$ & 7 & $D_{(7, k)}$ \\
$\mathrm{XCT}_{\mathrm{L}}$ & Hough & 2 & 4 & $D_{(2,4, k)}$ & 8 & $D_{(8, k)}$ \\
$\mathrm{OM}$ & Hough & 2 & 5 & $D_{(2,5, k)}$ & 9 & $D_{(9, k)}$ \\
$\mathrm{SEM}$ & Hough & 2 & 6 & $D_{(2,6, k)}$ & 10 & $D_{(10, k)}$ \\
\hline
\end{tabular}

For the three-way layout we consider the measured diameter for fibre $k$, algorithm $i$ and mode $j$,

$$
\text { Model I: } \begin{aligned}
D_{i j k}=\mu & +\alpha_{i}+\beta_{j}+(\alpha \beta)_{i j}+\gamma_{k}+\epsilon_{i j k}, \\
& \quad i=1,2, j=1, \ldots, 6 \text { and } k=1, \ldots, N
\end{aligned}
$$

where $\mu$ is the general level, $\alpha_{i}$ is the deviation from the mean for algorithm $i$, $\beta_{j}$ is the deviation from the mean for mode $j,(\alpha \beta)_{i j}$ is the interaction between algorithm and mode, and $\gamma_{k}$ is the $k$ 'th fibre's deviation from the mean. The error terms $\epsilon_{i j k}$ are assumed to be independent and normally distributed with the same variance, i.e. $\epsilon_{i j k} \sim \mathcal{N}\left(0, \sigma^{2}\right)$. $N$ is the number of fibres. Note that we do not have measurements for all combinations of algorithm and mode since the 3D-based data analysis is not possible for the surface-based modes OM and SEM. 
For the two-way model we describe the measured diameters as

Model II: $\quad D_{\nu k}=\mu+\theta_{\nu}+\gamma_{k}+\epsilon_{\nu k}, \quad \nu=1, \ldots, 10$ and $k=1, \ldots, N$, with similar interpretations and assumptions as in Model I.

Until now, we ignored the spatial coordinates of the fibres but now we investigate the possible dependence on the spatial layout by considering regression on fibre coordinates, i.e. we fit polynomial models to the size of the diameter as a function of the centre coordinates $\left(x_{k}, y_{k}\right)$ for the $k^{\prime}$ th fibre. For the methods $\nu=1, \ldots, n$ we consider the models:

$$
\begin{aligned}
& \mathrm{A}: \quad D_{\nu k}=\gamma(\nu)+\delta(\nu) x_{k}+\rho(\nu) y_{k}+\eta(\nu) x_{k}^{2}+\kappa(\nu) y_{k}^{2}+\lambda(\nu) x_{k} y_{k}+\epsilon_{\nu k}, \\
& \mathrm{~B}: \quad D_{\nu k}=\gamma(\nu)+\delta x_{k}+\rho y_{k}+\eta x_{k}^{2}+\kappa y_{k}^{2}+\lambda x_{k} y_{k}+\epsilon_{\nu k}, \\
& \mathrm{C}: \quad D_{\nu k}=\gamma+\delta x_{k}+\rho y_{k}+\eta x_{k}^{2}+\kappa y_{k}^{2}+\lambda x_{k} y_{k}+\epsilon_{\nu k},
\end{aligned}
$$

235 where the errors are independent and normally distributed $\epsilon_{\nu k} \sim \mathcal{N}\left(0, \sigma^{2}\right)$. The first model corresponds to individual, quadratic surfaces, one for each method. In the second case we have the same coefficients to the spatial coordinates for all methods, only the intercepts $\gamma(\nu)$ are method-dependent. In the last case we use the same surface for all methods.

The unknown parameters in the models above are estimated using ordinary least squares, and hypothesis on possible simplifications of the models are done by means of suitable Analyses of Variance (ANOVA). For a detailed description see for instance [19.

Multivariate approach. In the multivariate approach, we organise the observations in ten-dimensional vectors

$$
D_{k}=\left[D_{1, k}, D_{2, k}, D_{3, k}, \ldots, D_{10, k}\right]^{T}, \quad k=1, \ldots, N
$$

Under the normality assumption, the multivariate distribution of the vectors $D_{k}$ is determined by the means and standard deviations of the coordinates (in this case measured diameters) in the observation vector defined above, and the correlations between those. The latter will be collected in the correlation matrix 


$$
Q=\left[\begin{array}{ccc}
1 & \cdots & \rho_{1,10} \\
\vdots & \ddots & \vdots \\
\rho_{1,10} & \cdots & 1
\end{array}\right],
$$

250 the $s^{\prime}$ th component in $D_{k}$. If we project $D_{k}$ on the eigenvectors of $Q$ we obtain the so-called principal components (PC) of $D_{k}$. They are uncorrelated and the first component describes the maximal variation amongst all linear combinations (with normed coefficients) of $D$-components. The following components the previous. Such a Principal Component Analysis (PCA) [20] is thus a decomposition of the often very correlated coordinates in the observation vector into independent components describing decreasing amounts of the total variation.

\section{Results and discussion}

The field of view varies between scans, which constraints the amount of fibres that can be compared. Our comparison is based on $N=757$ fibres. Fig. 2 shows the fibres seen in all scans, and the overlap between these images defines the region of interest where we have carried out the analysis. Only the fibres in the lower part of the image have been analysed (circled in red in Fig. 4 (a)), because these are from a UD bundle that is orthogonal to the viewing direction. The upper fibres (not circled) are backing fibres that are at an angle of $45^{\circ}$ relative to the UD part. This difference in orientation is easily seen in the upper part of the image in Fig. 3. Examples of the fibre segmentation are shown over the different scans in Fig. 4 .

Fig. 4 shows that most fibres are found quite precisely for all scans using both algorithms. The only exception is the low-resolution XCT analysed with the circular Hough transform, where some non-existent fibres are detected, as shown in Fig. 4(k). Since we focus on validation, we remove falsely detected fibres by utilising the alignment of images to only keep the fibres also found 


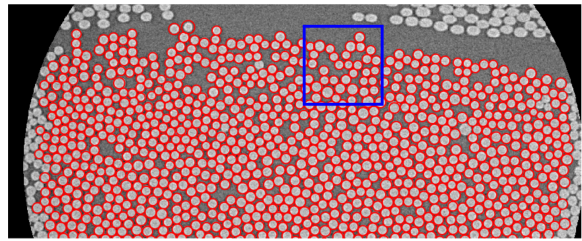

(a) $\mathrm{XCT}_{\mathrm{H}}$ with fibres detected.

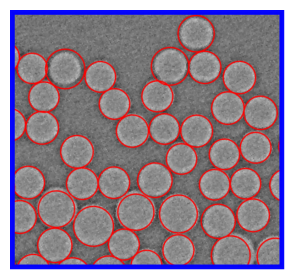

(d) SRCT Prob.

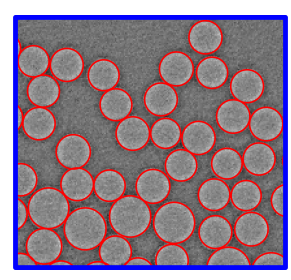

(h) SRCT Hough

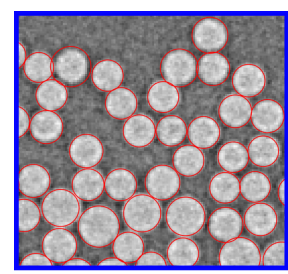

(e) $\mathrm{XCT}_{\mathrm{H}}$ Prob.

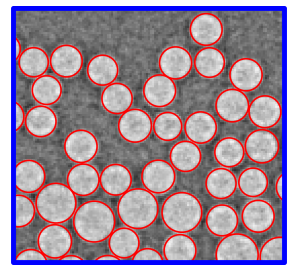

(i) $\mathrm{XCT}_{\mathrm{H}}$ Hough

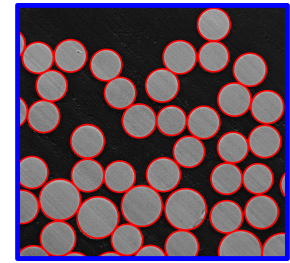

(b) SEM

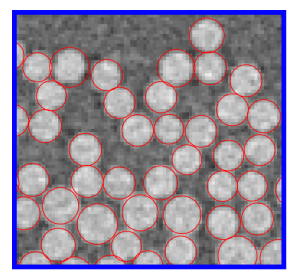

(f) $\mathrm{XCT}_{\mathrm{M}}$ Prob.

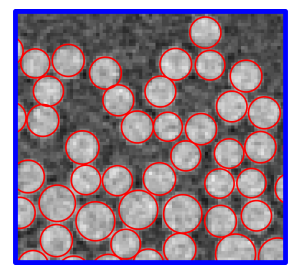

(j) $\mathrm{XCT}_{\mathrm{M}}$ Hough

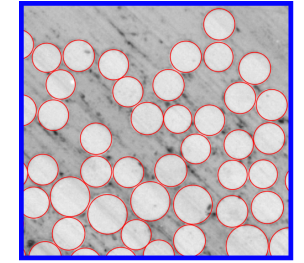

(c) $\mathrm{OM}$

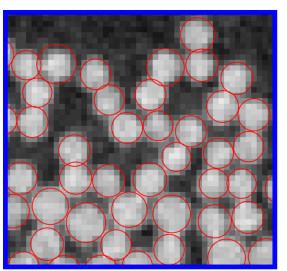

(g) $\mathrm{XCT}_{\mathrm{L}}$ Prob.

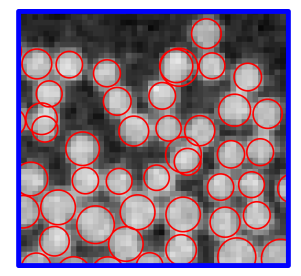

(k) $\mathrm{XCT}_{\mathrm{L}}$ Hough

Figure 4: Diameter estimates over scans. In (a) the diameters for the high-resolution XCT scan. In (b-k) zoomed versions of all scans showing the diameter estimates inside the blue square marked in (a) are shown. (k) shows some misdetected fibres using the Hough transform.

275 by other methods. It should be noted that the Hough transform algorithm would not be directly applicable for fibre segmentation at low resolutions when reference detections (obtained through other methods) are not available.

\subsection{Univariate approach}

The result of the three-way (Model I) and two-way (Model II) analyses presented in Section 3.2 showed a significant effect of all parameters, so there is significant difference between fibres, algorithm, mode, and the algorithm and mode in combination.

Average diameters and deviations from the mean for the two-way analysis (Model II) are given in Tab. 3. There are six groups of measurements, and 
Table 3: Multiple comparisons between the means $(\hat{\mu}+\hat{\theta})$ of the 10 methods using Model II. Average deviation is given by $\hat{\theta}_{\nu}$, and superscripts denote groups in which the average diameters are not significantly different based on Tukey's multiple comparison tests in a twoway analysis of variance over Model II. The overall mean is $\hat{\boldsymbol{\mu}}=16.57 \mu \mathrm{m}$.

\begin{tabular}{lllll}
\hline $\begin{array}{l}\text { Algorithm } \\
\text { Mode }\end{array}$ & Mean: $\hat{\mu}+\hat{\theta}_{\nu}$ & Deviation: $\hat{\theta}_{\nu}$ & Mean: $\hat{\mu}+\hat{\theta}_{\nu}$ & Deviation: $\hat{\theta}_{\nu}$ \\
\hline $\mathrm{SEM}$ & $17.05 \mathrm{\mu m}^{\mathbf{a}, \mathbf{b}}$ & $0.48 \mu \mathrm{m}$ & - & - \\
$\mathrm{OM}$ & $16.55 \mathrm{~mm}^{\mathbf{d}}$ & $-0.027 \mu \mathrm{m}$ & - & - \\
$\mathrm{SRCT}$ & $16.86 \mathrm{~m}^{\mathbf{c}}$ & $0.29 \mu \mathrm{m}$ & $17.15 \mu^{\mathbf{a}}$ & $0.57 \mu \mathrm{m}$ \\
$\mathrm{XCT}_{\mathrm{H}}$ & $16.32 \mathrm{\mu m}^{\mathbf{e}}$ & $-0.25 \mu \mathrm{m}$ & $17.06 \mu^{\mathbf{a}, \mathbf{b}}$ & $0.48 \mu \mathrm{m}$ \\
$\mathrm{XCT}_{\mathrm{M}}$ & $16.26 \mu \mathrm{m}^{\mathbf{e}}$ & $-0.32 \mu \mathrm{m}$ & $16.96 \mathrm{~m}^{\mathbf{b}, \mathbf{c}}$ & $0.39 \mu \mathrm{m}$ \\
$\mathrm{XCT}_{\mathrm{L}}$ & $14.96 \mu \mathrm{m}^{\mathbf{f}}$ & $-1.62 \mu \mathrm{m}$ & $16.58 \mathrm{~mm}^{\mathbf{d}}$ & $0.0026 \mu \mathrm{m}$ \\
\hline
\end{tabular}

within each of these groups the estimated means are not significantly different. Fibre diameter estimates for groups $\mathbf{a}, \mathbf{b}$ and $\mathbf{c}$ are very close to the product specification of $17 \mu \mathrm{m}$, and the high-resolution SEM is in this group. Diameters estimated from OM are on average $0.50 \mu \mathrm{m}$ smaller than SEM, despite OM and SEM having comparable pixel sizes. For the micro-CT modes, the diameter estimates decrease with lower resolution, and this is especially pronounced when using the circular Hough transform. Here the estimate from low resolution (classified in group $\mathbf{f}$ ) is significantly different from all other estimates. A smaller decrease is observed using the probabilistic feature labelling algorithm, where only the low resolution is significantly different from SEM. Probabilistic feature labelling also gives very consistent standard deviations independently of scan resolution.

\section{Spatial distributions of diameters}

For analysing the three spatial models presented in Section 3.2 we reduced the set of fibres further, because least square estimates are sensitive to extreme outliers. Therefore, we made an outlier test on the $6 \times 757$ fibre measurements 
using the studentised residuals from a simple two-sided ANOVA with fibres and methods as factors. Residuals with an absolute value larger than $3.5 \mu \mathrm{m}$ were considered extreme and discarded from the analysis, leaving 738 fibres for further statistical investigations. The simplification from model A to model B 


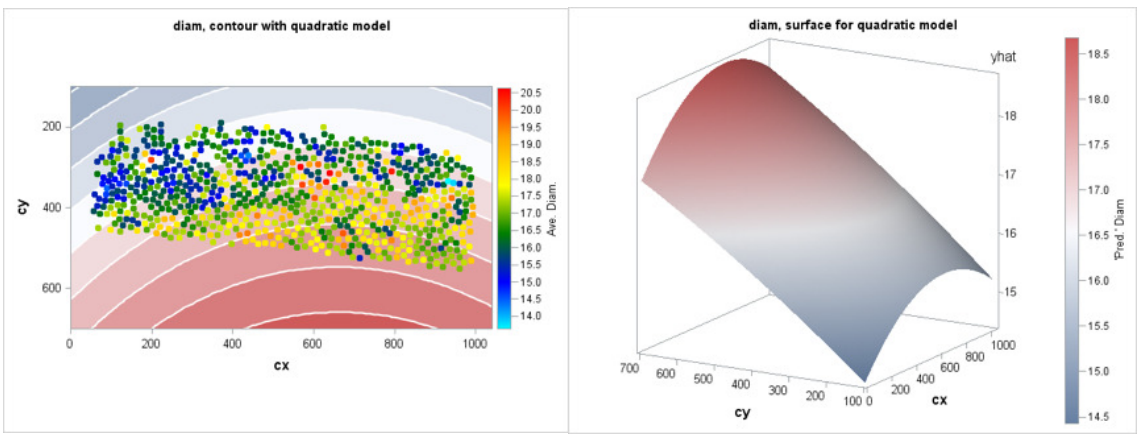

(a)

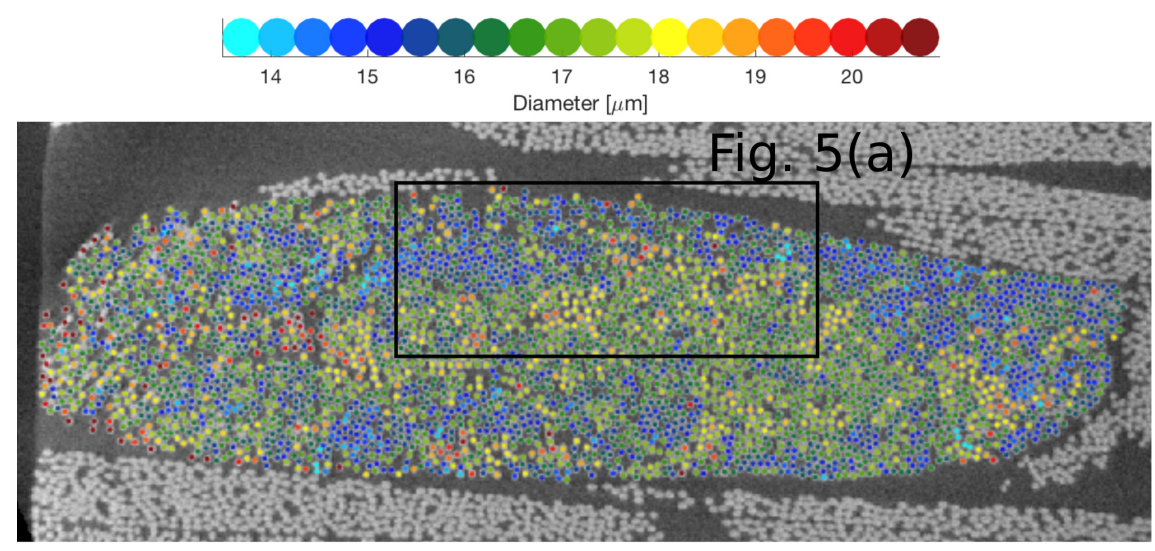

(b)

Figure 5: Spatial distribution of diameter values. In (a) measured and predicted diameters inside the RoI. On the left, individual fibres are coloured according to the average of the diameter estimates across six methods. Contour lines (left) and surface (right) for the quadratic fit in model C (based on $N=738$ fibres) give the predicted diameter values. In (b) diameters measured for the whole UD bundle from the low-resolution XCT mode with our Prob. algorithm.

terial under transverse loading 21. If combined with the 3D fibre trajectories, the longitudinal stiffness and the compression strength of the measured microstructure can also be computed through finite element modelling. Moreover, the formula by Budiansky [22] can be employed to estimate the compression strength from the measured fibre orientations [8] and, through Krenchel's formula [23, a fibre orientation efficiency factor can be estimated based on the 
individual fibre orientations to estimate the longitudinal stiffness of the sample.

\subsection{Multivariate approach}

340 resolution modes (SEM, OM, SRCT and $\mathrm{XCT}_{\mathrm{H}}$ ). The plot is coloured according to three groups, i.e. the SEM and OM (blue), $\mathrm{XCT}_{\mathrm{H}}$ and $\mathrm{SRCT}$ using the circular Hough transform (green) and the probabilistic feature labelling (red). The interactions between groups are shown in other colours. It can be seen that OM

Table 4: Principal components based on the covariance of high-resolution modes illustrated in Fig. 6

\begin{tabular}{lcccccc}
\hline \multirow{2}{*}{ Alg. } & \multicolumn{3}{c}{ Hough } & & \multicolumn{2}{c}{ Prob. } \\
Mode & OM & SEM & SRCT & XCT $_{\mathrm{H}}$ & SRCT & XCT $_{\mathrm{H}}$ \\
\hline PC1 & 0.4139 & 0.4178 & 0.4186 & 0.4130 & 0.3955 & 0.3898 \\
PC2 & -0.2977 & -0.2827 & -0.2825 & -0.2461 & 0.5649 & 0.6099 \\
\hline
\end{tabular}

A comparison of the micro-CT modes for each of the two algorithms is shown in Fig. 7. The plot is divided into a blue part showing results obtained with the probabilistic feature labelling, red showing results based on the circular Hough 


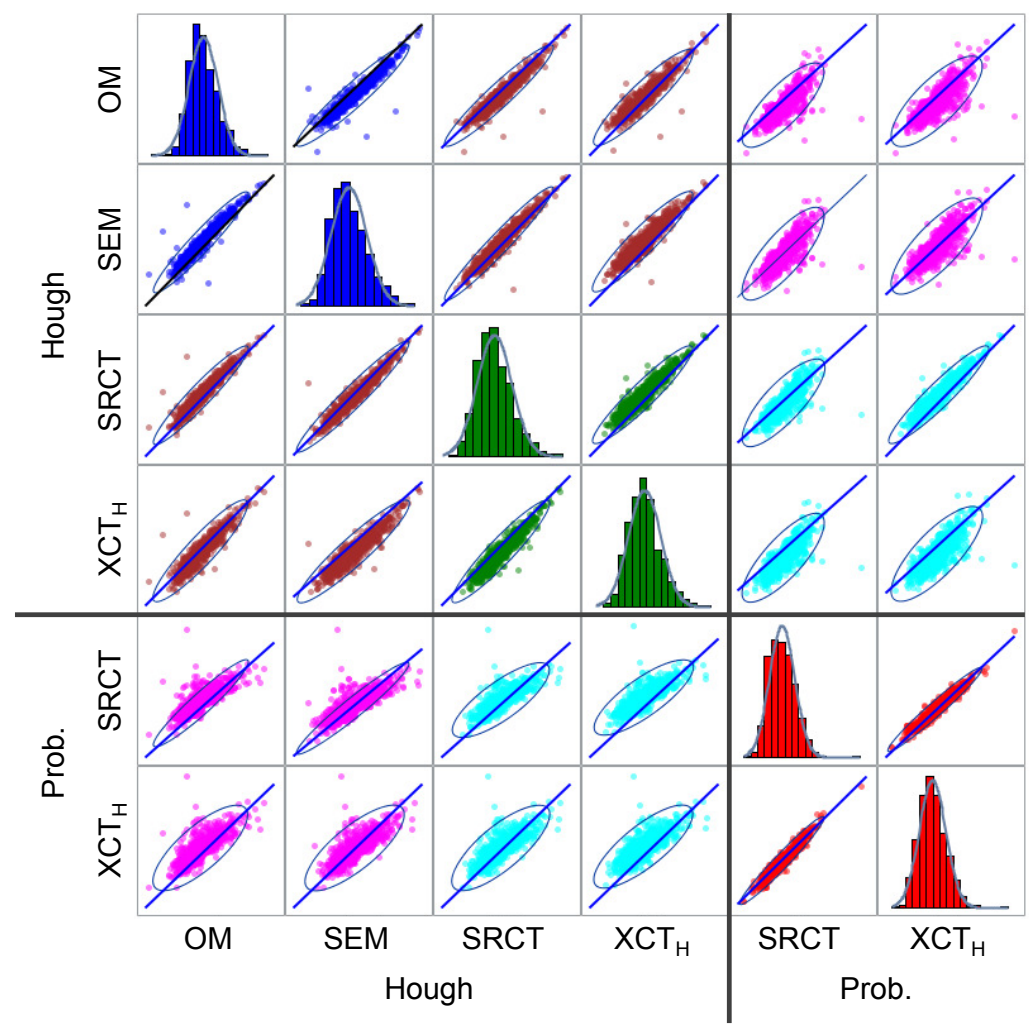

Figure 6: Scatter plots for diameters obtained for the high-resolution modes. Methods employed row-wise are OM, SEM, SRCT, and $\mathrm{XCT}_{\mathrm{H}}$, first with the circular Hough transform and then with the probabilistic feature labelling. The blue line is the identity line and the blue ellipse is the $99 \%$ prediction ellipse for a new observation.

transform, and magenta showing the interactions. Again, it is evident that the spread within groups is smaller than between groups, and especially the lowresolution XCT mode analysed with the circular Hough transform deviates very much from the other methods.

The principal components in Tab. 55 show again that the first principal component is approximately proportional to the mean of all methods and that the second principal component captures the difference between the algorithms. Furthermore, it can be seen that the coefficient for the Hough algorithm over $\mathrm{XCT}_{\mathrm{L}}$ deviates considerably from the others. This corresponds to the rather 


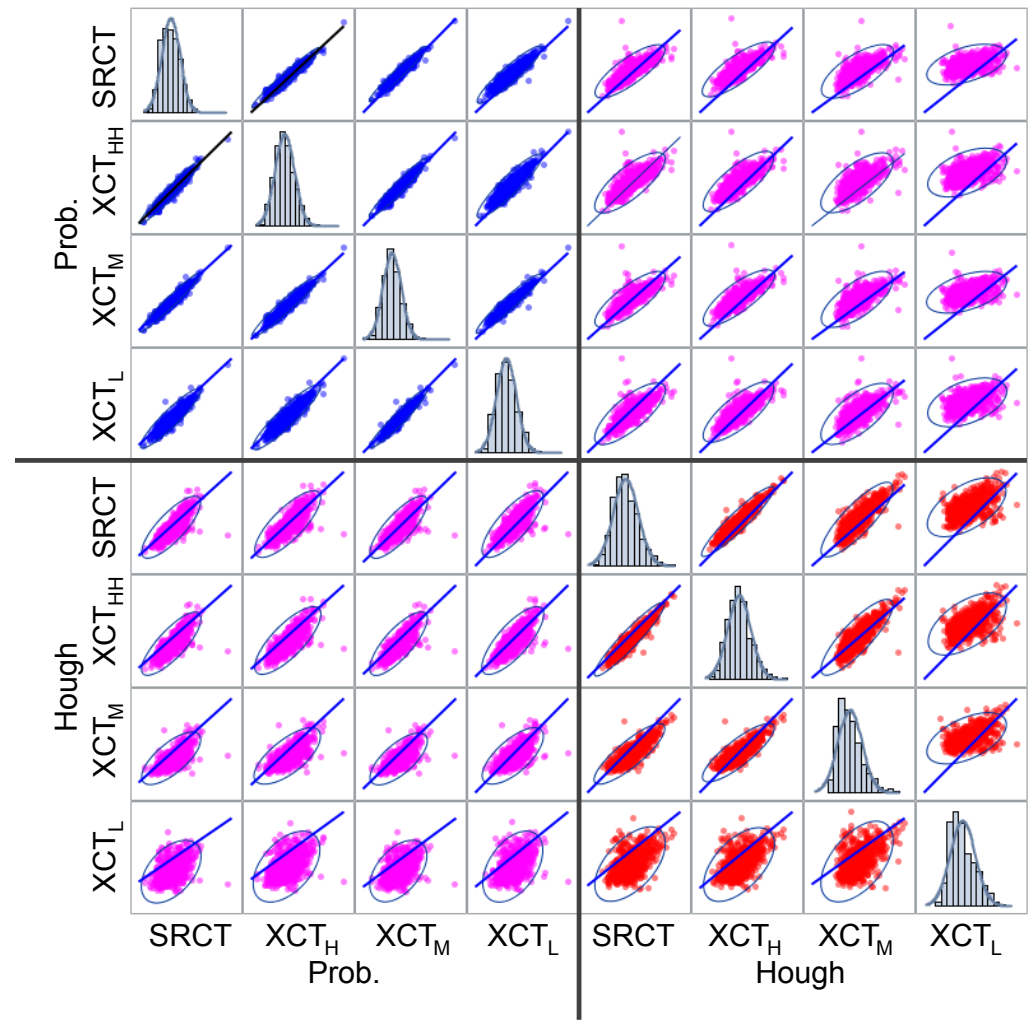

Figure 7: Scatter plots for diameters obtained with micro-CT imaging. Methods employed row-wise are SRCT, and high-, mid-, and low-resolution XCT, first with Hough transform and then with probabilistic feature labelling. The blue line is the identity line and the blue ellipse is the $99 \%$ prediction ellipse for a new observation.

low correlation between values obtained with the Hough transform and with the remaining methods, as mentioned when discussing Fig. 7.

A reasonable conclusion drawn from the statistical analysis is that we have reached the resolution limit for obtaining reliable results with the Hough transform algorithm, while the probabilistic labelling still provides results consistent with high-resolution images. The probabilistic algorithm is able to deal with low-resolution scans because it is based on learning a dictionary from the data at hand, which makes the algorithm flexible with respect to image contrast and resolution. Instead, the Hough algorithm relies on well-defined edges. For this 
reason, the results given by the latter algorithm degrade fast as fibre boundaries become unclear to due pixellation.

Table 5: Principal components based on the covariance of micro-CT methods illustrated in Fig. 7

\begin{tabular}{|c|c|c|c|c|c|c|c|c|}
\hline \multirow{2}{*}{$\begin{array}{l}\text { Alg. } \\
\text { Mode }\end{array}$} & \multicolumn{4}{|c|}{ Prob. } & \multicolumn{4}{|c|}{ Hough } \\
\hline & $\mathrm{SRCT}$ & $\mathrm{XCT}_{\mathrm{H}}$ & $\mathrm{XCT}_{\mathrm{M}}$ & $\mathrm{XCT}_{\mathrm{L}}$ & SRCT & $\mathrm{XCT}_{\mathrm{H}}$ & $\mathrm{XCT}_{\mathrm{M}}$ & $\mathrm{XCT}_{\mathrm{L}}$ \\
\hline PC1 & 0.3777 & 0.3742 & 0.3777 & 0.3686 & 0.3650 & 0.3647 & 0.3352 & 0.2452 \\
\hline $\mathrm{PC} 2$ & -0.2589 & -0.2642 & -0.2825 & -0.2999 & 0.1932 & 0.1910 & 0.2777 & 0.7365 \\
\hline
\end{tabular}

\section{Conclusion}

Our investigation demonstrates that X-ray CT combined with an adequate image analysis algorithm may have similar precision as scanning electron microscopy (SEM) and optical microscopy (OM) for characterising fibre composites based on diameters and centre positions, while it provides a non-destructive characterisation in 3D. We also showed that high precision can be obtained even if the resolution is lowered significantly, and we obtained results similar to SEM with X-ray CT images at nine times lower resolution and only a slight degradation in performance at fifteen times lower resolution. However, this performance requires high precision methods for fibre segmentation, such as our probabilistic feature labelling method. This illustrates that when using image-based characterisation it is important to consider the imaging modality in combination with the image analysis method. In addition to high precision, X-ray CT combined with the proposed segmentation method opens the possibility for high precision analysis of large volumes, which has a great potential in giving insights for understanding the relation between fibre micro-structure and material properties. 


\section{Acknowledgement}

Financial support from CINEMA: "the allianCe for ImagiNg of Energy MAterials", DSF-grant no. 1305-00032B under "The Danish Council for Strategic Research" is gratefully acknowledged. The staff at ID19, beamline of the European Synchrotron Research Facility, is acknowledged for kindly performing the scanning. A special thanks to Alexander Rack and Vincent Fernandez.

\section{References}

[1] R. Pyrz, Application of X-ray microtomography to the study of polymer composites, Tech. rep., SAE Technical Paper (1999).

[2] G. Requena, G. Fiedler, B. Seiser, P. Degischer, M. Di Michiel, T. Buslaps,
3D-quantification of the distribution of continuous fibres in unidirectionally reinforced composites, Composites Part A: Applied Science and Manufacturing 40 (2) (2009) 152-163.

[3] J. E. Little, X. Yuan, M. I. Jones, Characterisation of voids in fibre reinforced composite materials, NDT \& E International 46 (2012) 122-127.

[4] M. J. Emerson, K. M. Jespersen, P. S. Jørgensen, R. Larsen, A. B. Dahl, Dictionary based segmentation in volumes, in: Scandinavian Conference on Image Analysis, Springer, 2015, pp. 504-515.

[5] A. Reh, B. Plank, J. Kastner, E. Gröller, C. Heinzl, Porosity mapsinteractive exploration and visual analysis of porosity in carbon fiber reinforced polymers, in: Computer Graphics Forum, Vol. 31, Wiley Online Library, 2012, pp. 1185-1194.

[6] K. K. Kratmann, M. Sutcliffe, L. Lilleheden, R. Pyrz, O. T. Thomsen, A novel image analysis procedure for measuring fibre misalignment in unidirectional fibre composites, Composites Science and Technology 69 (2) (2009) 228-238. 
[7] Z. Yang, W. Ren, M. Mostafavi, S. A. Mcdonald, T. J. Marrow, Characterisation of 3D fracture evolution in concrete using in-situ X-ray computed tomography testing and digital volume correlation, in: VIII International Conference on Fracture Mechanics of Concrete and Concrete Structures, 2013, pp. 1-7.

[8] M. J. Emerson, K. M. Jespersen, A. B. Dahl, K. Conradsen, L. P. Mikkelsen, Individual fibre segmentation from 3d x-ray computed tomography for characterising the fibre orientation in unidirectional composite materials, Composites Part A: Applied Science and Manufacturing 97 (2017) 83-92.

[9] D. Tsarouchas, A. Markaki, Extraction of fibre network architecture by X-ray tomography and prediction of elastic properties using an affine analytical model, Acta Materialia 59 (18) (2011) 6989-7002.

[10] D. Bull, L. Helfen, I. Sinclair, S. Spearing, T. Baumbach, A comparison of multi-scale 3D X-ray tomographic inspection techniques for assessing carbon fibre composite impact damage, Composites Science and Technology 75 (2013) 55-61.

[11] J. Kastner, B. Plank, D. Salaberger, J. Sekelja, Defect and porosity determination of fibre reinforced polymers by X-ray computed tomography, in: 2nd International Symposium on NDT in Aerospace, 2010, pp. 1-12.

[12] Y. Nikishkov, L. Airoldi, A. Makeev, Measurement of voids in composites by X-ray computed tomography, Composites Science and Technology 89 (2013) 89-97.

[13] M. W. Czabaj, M. L. Riccio, W. W. Whitacre, Numerical reconstruction of graphite/epoxy composite microstructure based on sub-micron resolution X-ray computed tomography, Composites Science and Technology 105 (2014) 174-182. 
[14] R. Sencu, Z. Yang, Y. Wang, P. Withers, C. Rau, A. Parson, C. Soutis, Generation of micro-scale finite element models from synchrotron X-ray CT images for multidirectional carbon fibre reinforced composites, Composites Part A: Applied Science and Manufacturing 91 (2016) 85-95.

[15] A. L. Dahl, R. Larsen, Learning dictionaries of discriminative image patches, in: BMVC, 2011, pp. 1-11.

[16] K. M. Jespersen, L. P. Mikkelsen, Three dimensional fatigue damage evolution in non-crimp glass fibre fabric based composites used for wind turbine blades, Composites Science and Technology 153 (2017) 261-272.

[17] T. J. Atherton, D. J. Kerbyson, Size invariant circle detection, Image and Vision computing 17 (11) (1999) 795-803.

[18] H. Yuen, J. Princen, J. Illingworth, J. Kittler, Comparative study of hough transform methods for circle finding, Image and vision computing 8 (1) (1990) 71-77.

[19] D. C. Montgomery, Design and analysis of experiments, John Wiley \& Sons, 2017.

[20] R. A. Johnson, D. W. Wichern, et al., Applied multivariate statistical analysis, Vol. 4, 2014.

[21] L. P. Mikkelsen, M. J. Emerson, K. M. Jespersen, V. A. Dahl, K. Conradsen, A. B. Dahl, X-ray based micromechanical finite element modeling of composite materials, in: 29th Nordic Seminar on Computational Mechanics Nordic Seminar on Computational Mechanics, 2016.

[22] B. Budiansky, N. Fleck, Compressive failure of fibre composites, Journal of the Mechanics and Physics of Solids 41 (1) (1993) 183-211.

[23] H. Krenchel, Fibre reinforcement; theoretical and practical investigations of the elasticity and strength of fibre-reinforced materials, Alademisk forlag, 1964. 